\title{
Animated Subject Maps for Book Collections
}

\section{Tim Donahue}

ABSTRACT

Of our two primary textual formats, articles by far have received the most fiscal and technological support in recent decades. Meanwhile, our more traditional format, the book, seems in some ways to already be treated as a languishing symbol of the past. The development of OPACs and the abandonment of card catalogs in the 1980s and 1990s is the seminal evolution in print monograph access, but little else has changed. To help users locate books by call number and browse the collection by subject, animated subject maps were created. While the initial aim is a practical one, helping users to locate books and subjects, the subject maps also reveal the knowledge organization of the physical library, which it displays in a way that can be meaningful to faculty, students, and other community members. We can do more with current technologies to assist and enrich the experience of users searching and browsing for books. The subject map is presented as an example of how we can do more in this regard.

\section{LC CLASSIFICATION, BOOKS, AND LIBRARY STACKS}

During the last few decades of technological evolution in libraries, we have helped facilitate a seismic shift from print-based to digital research. Our library websites are jammed with electronic resources, digital collection components, database links, virtual reference assistance, online tutorials, and mobile apps. Collection budgets too have shifted from a print to electronic focus. Many libraries are now spending less than 20 percent of their material budgets on print monographs. And yet, our stacks are still filled with books that often take up more than fifty percent of our library spaces. Knowledge organization schemas have also evolved in libraries. We have subject lists to help users to decide on which databases to select that reflect current disciplines and majors in higher education. Internal database navigation continues to evolve in terms of limits, fields, and subject searching. Web searching is based on the contemporary keyword approach where "everything is miscellaneous" and need not be organized, but nationwide, billions of books still sit on shelves according to Dewey or Library of Congress classification systems that were initially developed over a century ago. Some say these organizing systems are wo efully antiquated and do not reflect our contemporary post-modern realities, though they still amply serve their purpose to assign call number locations for our books. We hear scant little of plans to update these classification schemes. Why invest more time, energy, and resources on revamped organization schemes for libraries? The HathiTrust now contains the

Tim Donahue (tdonahue@montana.edu) is Assistant Professor/Instruction Librarian, Montana State University, Bozeman, MT. 
scanned text of more than ten million books. Google claims there are almost 130 million published titles in the world and intends to digitize all of them. ${ }^{1}$ What will happen to our physical book collections? How long will they reside on our library shelves? How long will they be located using the Dewey and LC systems? Is the library a shrinking organism?

Profession-wide, there seems to be no concrete vision in regards to the future of our book collections. There is, of course, general acknowledgement that acquisition of e-books will increase as print acquisitions decrease and that, overall, print collections will accordingly shrink to reflect the growing digital nature of knowledge consumption. But for now and into the foreseeable future these billions of monographs remain on our shelves in the same locations our call number systems assigned to them decades ago. And while online library users are now able to utilize an array of electronic access delivery systems and web technologies for their article research and consumption, book seekers still need a call number.

Books and articles have been our two primary textual formats for centuries. Articles have moved into the digital realm more fleetly than their lengthier counterparts. Their briefer length, the cyclical serial publication process, and the evolution of database containment and access have enabled, in a relatively short time, a migration from print to primarily digital access. Books, however, are accessed in much the same way they were a hundred years ago. The development of OPACs in the 1980s and 1990s and abandonment of card catalogs is the seminal evolution in print monograph access, but little else has changed. ${ }^{2}$ Once a call number is attained, the rest of the process remains physical, usually requiring pencil, paper, feet, sometimes a librarian, and a trip through the library until the object itself is found and pulled from the shelf. So while the process of article acquisition may employ a plethora of finding aids, keyword searching, database features, full text availability, and various delivery methods through our richly developed websites, beyond the OPAC and possibly a static online map, book seekers are on their own or need a librarian in what may seem a meaningless labyrinth of stacks and shelves.

While the primary and most practical purpose of our classification schemes is to provide an assigned call number for book finding, these organizational outlines create an order to the layout of our stacks that maps a universe of knowledge within our library walls. This structure of knowledge reveals a meaning to our collections that includes the colocation of books by topic and proximity of related subjects. These features enhance the browsing process and often lead to the act of serendipitous discovery.

To locate a book by call number, a user may consult library floor plans, which are typically limited to broad ranges or LC main classes, then rely on stack-end cards to home in on the exact stack location. To browse books by subject without using the catalog, a user typically must rely on a combination of floor plans and LC Outline posters if they exist at all. Often, informed browsing by subject cannot take place without a visit to the reference desk for mediation by a librarian. Even then, many librarians are barely familiar with their book collection's organizational structure and are reticent to recommend broad subject browsing. 


\section{PURPOSE AND DESCRIPTION OF THE SUBJECT MAP}

To help users locate books by call number and browse the collection by subject, animated subject maps were created atSkidmore College and Montana State University. Displaying overhead views of library floors, users mouse over stacks to reveal the LC sub-classes located within. Alternatively, they may browse and select LC subject headings to see which stacks contain them. The LC Outline contains 21 main subject classes and 224 sub-classes, corresponding to the first two elements of a book call number. On stack mouse-over, three items are displayed: the call number by range, the main subject heading, and all sub-classes contained within the stack. When using the browse by subject option, users select and click an LC main class and the stacks where this class is located are highlighted. While the initial aim is a practical one, helping users to locate books and subjects, the subject map also reveals the knowledge organization of the physical library, which it displays in a way that can be meaningful to faculty, students, and other community members. The map also provides local electronic access to the LC Classification Outline. At both institutions the maps are linked from prominent web locations and electronic points of need that are relevant and proximate to other book searching functions and tools.

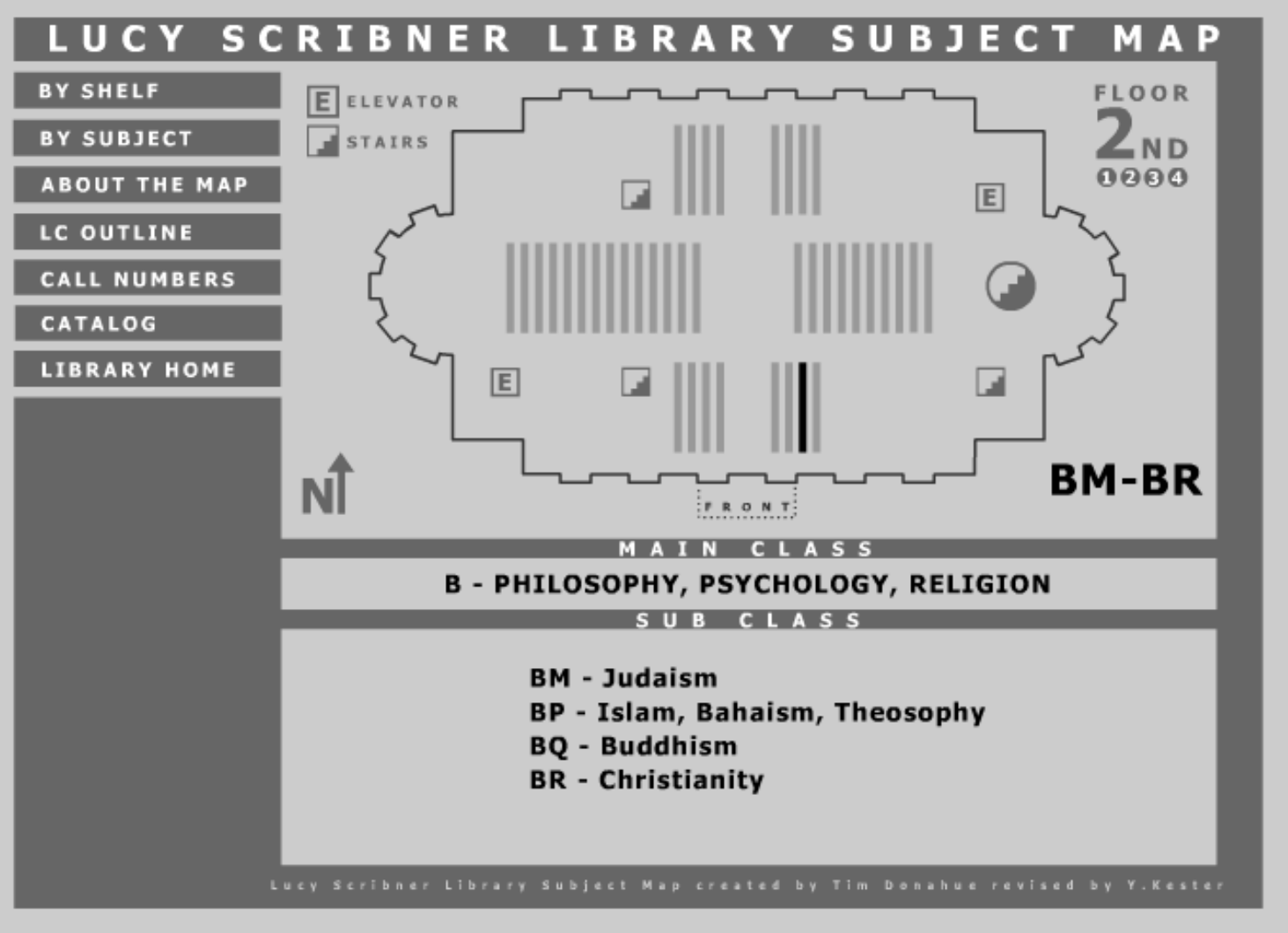

Figure 1. Skidmore College subject map showing stack mouse-over display. 


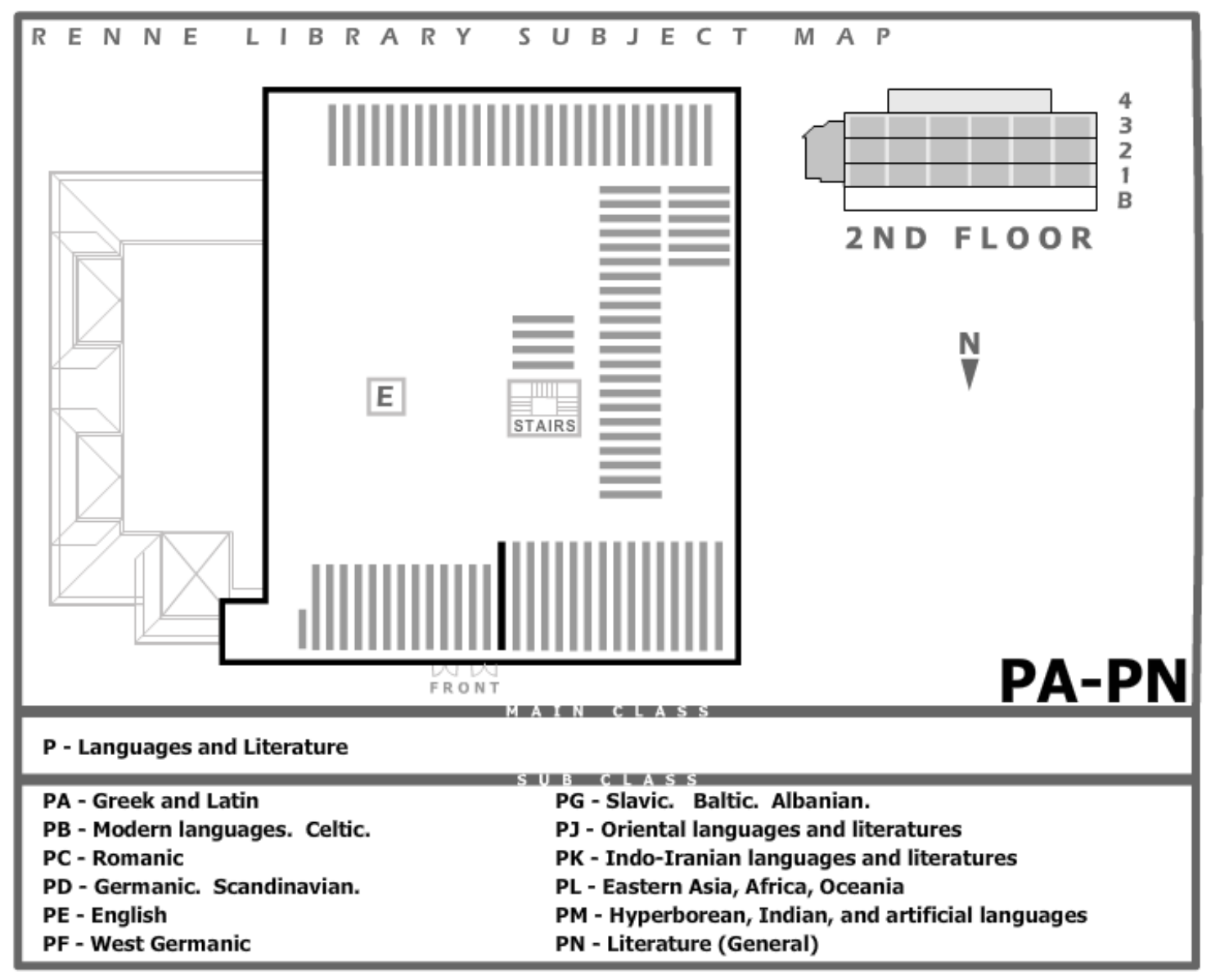

Figure 2. Montana State University subject map showing stack mouse-over display.

\section{DESIGN RATIONALE AND METHODOLOGY}

The inspiration for the subject map started with a question: What if users could see on a map where individual subjects were located within the library? Most library maps examined were limited to LC main classes or broad ranges denoting wide swaths of call numbers. Including hundreds of LC subclasses would convolute and clutter a floor map beyond usability. But what if an online map contained each individual stack and only upon user-activation was the information revealed, saving space and avoiding clutter? Such a map should be as devoid of congestion as possible and focus the user's attention on library stack locations and LC Classification.

Working from existing maps and architectural blueprints of the library building, a basic perimeter was rendered using Adobe Illustrator and InDesign software. These perimeters were then imported into Adobe Flash and a new .FLA file created. Library stacks were then measured, counted, and added as a separate layer within each floor perimeter. Basic location elements such as stairways, elevators, and doors were added for locational reference points. Each stack was then programmed as a button with basic rollover functionality. Flash ActionScript was coded so that the correct call number, main class, and sub-class information appear within the interface upon rollover activation. This functionality accounts for the stack searching ability of the subject map. 
Additionally, the LC outline was made searchable within the map so that users can mouse over subjects and upon clicking, see what stacks contain those main classes. This functionality accounts for the subject searching ability of the map. Left-hand navigation was built in so users can toggle between these two main search functions. Maintaining visual minimalism and simplicity was a priority and inclinations to render the map more comprehensively were resisted in order to maximize attention to subject and stack information. Black, white, and gray colors were chosen to enhance the contrast of the map and aid the user's eye for quick and clear use. Other relevant links and instructional context were added to the left-hand navigation including links to the Catalog, Official LC Outline, and library homepage. Finally, after uploading to the local server and creating a simple URL, links to the subject map were established in prominent and meaningful points of need within the library website.

\section{USER ACCEPTANCE}

Once the subject map was completed and links to it were made public, a brief demonstration was provided for reference team members who began showing it to users at the reference desk. Initial reaction was enthusiastic. Students thought it was "cool" and enjoyed "playing with it." One reported, "I didn't know the library actually made sense like that. It's neat to see the logic about where things are." Another student said, "Now I can see where all the books on Buddhism are!" Faculty, too, were pleased. Though faculty members typically know a little about LC Classification, they are not accustomed to seeing it visualized and grafted onto their institutional library's stacks. Making transparent the intellectual organization of the library for other faculty can bolster their confidence in our order and structure. Professors are often pleased to see their discipline's place within our stacks and where related subjects are located. The most positive praise for the subject map, however, comes from the sense of convenience it lends. Many comments express appreciation for the ability to directly locate an individual book stack. Because primary directional and finding elements like stairs and elevators are included in the maps, users are able to see the exact path that leads to the book they are seeking. For those not interested in browsing, in a hurry, or challenged in terms of mobility, the subject map is a time and energy saver. Some users however have reported frustration with the sensitivity required for the mouse-over functions. Others desire a more detailed level of searching beyond the sub-class level. One user pointed out that the subject map was of no help to the blind.

\section{MULTIPLE USES AND INTERNAL APPLICATIONS}

The primary use and most obvious application of the subject map is as a reference tool. As a front line finding aid, librarians and other public service staff at reference, circulation, or other help desks can easily and conveniently deploy the map to point users in the right direction and orient them to the book collection. In library instruction sessions, the subject map is not only a practical local resource worth pointing out, but also serves as an example of applied knowledge organization. When accompanying a demonstration of the library catalog, the map is not only a valuable finding aid, but adds a layer of meaning as well. Students who understand the map are 
not only more able to browse and locate books, but learn that a call number represents a detailed subject meaning as well as locational device. Used in conjunction with a tour, the map reinforces the layout of library shelves and helps to bridge the divide between electronic resources and physical retrieval. The subject map facilitates a concrete and visual introduction to the LC Classification Outline, a knowledge of which can be applied to most college and research libraries in the United States. The subject map can also be of assistance with Collection Development. Perusal of the map can reveal relative strengths and weaknesses within the collection. Subject liaisons and bibliographers may use the map to home in on and visualize their assigned areas. Circulation staff and stacks maintenance workers find the map useful for book retrieval, shifting projects, and in the training and acclimation of new workers to the library. The subject map has proven to be a useful reference for library redesign and space planning considerations. At information fairs and promotional events where devices or projection screens are available, the map has served as a talking point and promotional piece of digital outreach. The map has been demonstrated by information science professors to LIS graduate students as an example of applied knowledge organization in libraries. Recently, a newly hired incoming library dean commented that the map helped him "get to know the book collection" and familiarized him with the library.

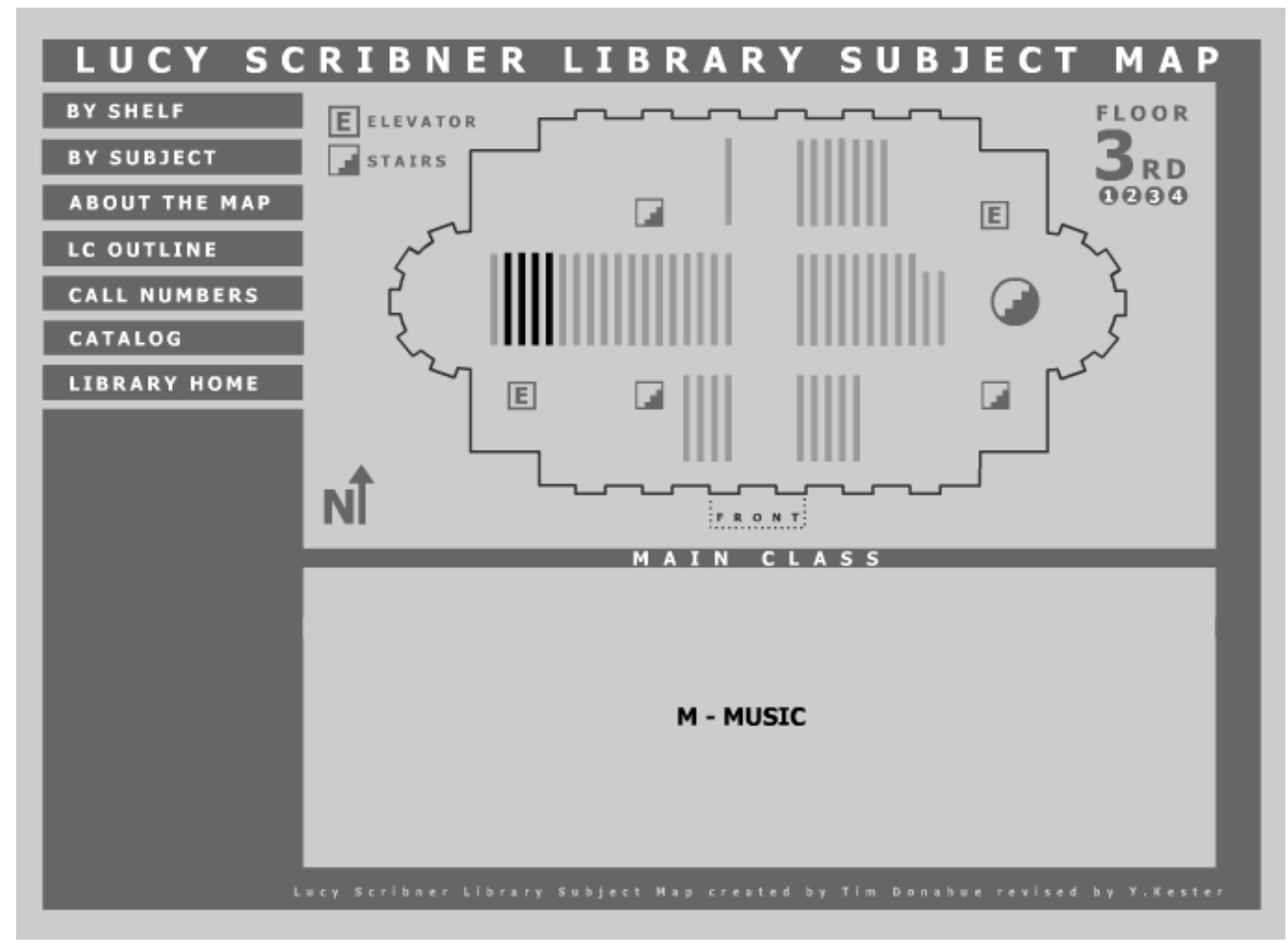

Figure 3. Skidmore College subject map showing subject search display. 


\section{ISSUES AND CHALLENGES}

In some libraries, books don't move for decades. The same subjects may reside on the same shelves during an entire library's lifetime. In this case, a subject map can be designed once and never edited. But, of course, most library buildings go through changes and evolutions. In many libraries, collection shifting seems to be ongoing. Book collections wax and wane. Certain subjects expand with their times, while others shrink in irrelevancy. Weeding does not affect all subjects and stacks equally and adjustments to shelves and end cards are necessary. In addition to the transitions of weeding and shifting, sometimes whole floors are reconfigured. In the library commons era of the last few decades, substantial redesigns have been commonplace as book collections make way for computer stations and study spaces. In all these cases, adjustments and updates will be necessary to keep a subject map accurate. This is easily done by going back into the master .FLA file and editing as needed. In many cases only a stack or two need be adjusted, but in instances of major collection shifting some planning ahead may be necessary and more time allotted for redesign. Shifting can be a complex spatial exercise and it is difficult to predict where subjects will realign exactly. Subject map editing may have to wait until physical shifting is completed. It should be noted that each stack must be hand-coded separately. In libraries with hundreds of stacks this can seem a tedious and time-consuming design method.

Both subject maps rely on Adobe Flash animation technology. Flash is proprietary software, so the benefits of open source software cannot be utilized with subject maps at this time. Further, Abobe Flash Reader software must be installed on a computer for the subject map to render. This has almost never been a problem, however, as the Flash Reader is ubiquitous and automatically installed on most public and private machines upon initial boot up. Another concern, however, relating to Flash technology is human assets. Not every library has a Flash designer or even someone who can implement the most fundamental Flash capabilities. Flash is not hard to learn and the subject maps utilize only its most basic functionalities, but still, for some it remains a niche software and many libraries will not have the resources to invest.

Reaction, though, to the live subject maps and the rollover interactivity they provide, has been so positive that more fully integrated Flash maps have been proposed. Why not have all physical elements of the library incorporated into one Flash-enabled map? This is possible but may come at some expense to the functionality of the subject-rendering aspect of the maps. By limiting the application to stacks and LC classes, a user may remain more focused. Avoiding clutter, overcrowding, and a preponderance of choice is a design strategy that has gained much credibility in recent years. ${ }^{3}$ The subject map enjoys the usability success of clean design, limited purpose, and simple rendering. While demonstrating the potential of user-activated animation for other proposed library applications, the subject map might be best maintained as a limited specialty map.

A final concern regarding the long-term success of subject maps should be mentioned. How long will books remain in libraries? How long will they be organized by subject? When the physical 
arrangement and organization of information objects no longer exists in libraries, maps of any kind will seemingly lose all efficacy. But will libraries themselves exist in this future? Whither books? Whither libraries?

\section{FUTURE DEVELOPMENTS}

The most prominent and practical attribute of the subject map is its ability to show a user the exact stack where the book they are seeking is located. But in its current state as a stand-alone application, a user must obtain a call number from a catalog search, then open the subject map by going to its independent URL. Investigation is underway to determine what is necessary in order to integrate the subject map with the online catalog. In this scenario, a catalog item record might also display an embedded subject map that auto matically highlights the floor and stack where the call number is located. This seemingly requires .SWF files and Flash ActionScript to be embedded in catalog coding. One potential solution is to attribute an individual URL to each stack rendering so that a get URL function can be applied and embedded in each catalog item record. This synthesis of subject map and catalog poses a complex challenge but promises meaningful and time-saving results for the item retrieval process.

QR code technology in conjunction with subject map use is also being deployed. By fixing QR codes on stack end cards that link to relevant sections of the LC Outline, a researcher may use a mobile device to browse digitally and physically within the stacks at the same time. In this way a user may conduct digital subject browsing and physical item browsing simultaneously. The URLs linked to by QR coding contain detailed LC sub-levels not contained within the subject map, which is limited to the level of sub-class. The active discovery of new knowledge facilitated by exploiting preexisting LC organization inside library stacks in real time can be quite impressive when experienced firsthand.

Another development exploiting LC knowledge organization is in beta mode at this time. An LC search database has been created allowing users to enter words and find matching LC subject terminology. Potentially, this database could be merged with the subject map, allowing users to correlate subject word search with physical locations independent of call numbers.

Despite its intent as a limited specialty map, possibilities are also being explored to incorporate the subject map into a more fully integrated library map. One way forward in this regard is to create map layers that could be toggled on and off by users. In this way, the subject map could exist as its own layer, maintaining its clarity and integrity when isolated but integrated when viewed with other layers. Flash technology excels at allowing such layer creation.

\section{OTHER STACK MAPS AND RELATED TECHNOLOGIES}

Searching the web for "subject map" and relative terminology such as stack, shelf, book, and LC maps, does turn up various efforts and approaches to organizing and exploiting classification scheme data, but no animated, user-activated maps are found. Similar searches across library and information science literature turn up some explorative research on the possibilities of mapping 
LC data, but again no animated stack maps are found. ${ }^{4}$ There is a product licensed by Bowker Inc. called StackMap that can be linked to catalog search results. When a user clicks on the map link next to a call number result, a map is displayed with the destination stack highlighted, but the information provided is locational only. StackMap is not animated or user-activated. No subject information is given and the map offers no browsing features.

Since the release of HTML5, we are beginning to see more animation on the web that is not Flashdriven. Steve Jobs and Apple's determined refusal to run Flash on their mobile devices has motivated many to seek other animation options. New HTML5 animation tools such as Adobe Edge, Hippo Animator, and Hype offer promising starts at dislodging the Flash grip on web animation, but they have far to go and do not yet offer either the ease of design nor the range of creative possibilities of Flash. Building an animated subject map with HTML5 alone does not seem possible at this time.

\section{UNIVERSAL APPLICABILITY OF THE SUBJECT MAP}

So far, subject maps have been created for two very different libraries. The commonality shared between the Montana State University and Skidmore College libraries is their possession of hundreds of thousands of books in stacks shelved by the LC classification system. This is a trait shared by nearly all college and research libraries. Subject maps can be easily structured on the Dewey Decimal System as well so that public libraries could benefit from their functionality, making the subject map appropriate and creatable for more than 12,000 libraries. ${ }^{5}$

Of our two primary textual formats, articles by far have received the most fiscal and technological support in recent decades. Article searching and retrieval continues to evolve through the rich implementation of assets such as locally constructed resource management tools, independent journal title searches, complexly designed database search interfaces, and dedicated electronic resource librarians. Meanwhile, our more traditional format, the book, seems in some ways to already be treated as a languishing symbol of the past. Because its future is uncertain, does that justify our neglect in the present? As a profession we seem a bit complacent about the state of our book collections. Why dedicate our technical resources to a format that is on the way out? But has the book disappeared yet? As we make room for more student lounges, coffee bars, computer stations, writing labs, and information commons, we should carefully ask what makes a library special. Good books and the focused, sustained treatment of knowledge they contain are part of the correct answer, symbolically and as yet, practically speaking. While our books still occupy our library shelves, shouldn't they also fully benefit from the ongoing technological explosion through which we continue to evolve? OPACs haven't evolved much in recent years. In fact they seem quite stymied to many librarians and users. We can do more with current technologies to assist and enrich the experience of users searching and browsing for books. The subject map is hopefully an example of how we can do more in this regard.

While we have grown accustomed to increasingly look forward in order to position our libraries for the future, we should also remember to sometimes look back. Our classification systems and 
book collections are assets built from the past that represent many decades of great labor, investment, and achievement. More than 12,000 public and academic libraries together make up one of our greatest national treasures and bulwarks of living democracy. Libraries are among the dearest valued assets in any of our states. Many of the most beautiful buildings in our nation are libraries. Based on library insurance values and estimated replacement costs, library buildings and the collections they hold amount cumulatively to hundreds of billions of dollars of worth. ${ }^{6}$ This astounding worth is figured mainly from the buildings themselves and the books they contain. A few have commented that there is some aesthetic quality to the subject maps. If this is true, the appeal comes from the synthesis of architectural form and the universe of knowledge revealed within, from the beauty of libraries both real and ideal, from physical and mental constructions unified. Animated subject maps can help bring the physical and intellectual beauty of libraries into the digital realm, but the main appeal is a practical one: to point the user directly to the book or subject they are seeking. So in conclusion, perhaps we should measure the subject map's potential in the light of Ranganathan's Five Laws of Library Science: ${ }^{7}$

1. Books are for use.

2. Every reader his [or her] book.

3. Every book its reader.

4. Save the time of the reader.

5. The library is a growing organism.

The subject maps can be found at the following URLs:

Skidmore College Subject Map:

http://lib.skidmore.edu/includes/files/SubjectMaps/subjectmap.swf

Montana State University Subject Map:

www.lib.montana.edu/subjectmap

\section{REFERENCES}

1. Google, "Google Books Library Project-An Enhanced Card Catalog of the World's Books," http://books.google.com/googlebooks/library.html, accessed November 8, 2012.

2. Antonella Iacono, "OPAC, Users, Web. Future Developments for Online Library Catalogues," Bollettino AIB 50, no. 1-2 (2010): 69-88, http://bollettino.aib.it/article/view/5296.

3. Geoffrey Little, "Where Are You Going, Where Have You Been? The Evolution of the Academic Library Web Site," The Journal of Academic Librarianship 38, no. 2, (2012): 123-25, doi:10.1016:j.acalib.2012.02.005. 
4. Kwan Yi and Lois Mai Chan, "Linking Folksonomy to Library of Congress Subject Headings: An Exploratory Study," Journal of Documentation 65, no. 6 (2009): 872-900, doi:10.1108:00220410910998906.

5. American Library Association, "Number of Libraries in the United States, ALA Library Fact Sheet 1," www.ala.org/tools/libfactsheets/alalibraryfactsheet01.

6. Edward Marman, "A Method for Establishing a Depreciated Monetary Value for Print Collections," Library Administration and Management 9, no. 2 (1995): 94-98.

7. S. R. Ranganathan, The Five Laws of Library Science (New Delhi: Ess Ess, 2006), http://hdl.handle.net/2027/mdp.39015073883822. 